Pacific

Journal of

Mathematics

DISTINGUISHED PRINCIPAL SERIES REPRESENTATIONS OF GL( $n$ ) OVER A $p$-ADIC FIELD

NADIR MATRINGE

Volume 239 No. 1

January 2009 


\title{
DISTINGUISHED PRINCIPAL SERIES REPRESENTATIONS OF GL(n) OVER A $p$-ADIC FIELD
}

\author{
NADIR MATRINGE
}

\begin{abstract}
Let $K / F$ be a quadratic extension of $p$-adic fields, and $\chi$ a character of $F^{*}$. A representation $(\pi, V)$ of $\operatorname{GL}(n, K)$ is said to be $\chi$-distinguished if there is a nonzero linear form $L$ on $V$ such that $L(\pi(h) v)=\chi \circ \operatorname{det}(h) L(v)$ for $h \in \mathbf{G L}(n, F)$ and $v \in V$. We classify here distinguished principal series representations of $\operatorname{GL}(n, K)$. Call $\eta_{K / F}$ the nontrivial character of $F^{*}$ that is trivial on the norms of $K^{*}$, and $\sigma$ the nontrivial element of the Galois group of $K$ over $F$. A conjecture attributed to Jacquet asserts that admissible irreducible representations $\pi$ of $\operatorname{GL}(n, K)$ are such that the smooth dual $\pi^{\vee}$ is isomorphic to $\pi \circ \sigma$ if and only if it is 1-distinguished or $\eta_{K / F^{-}}$ distinguished. Our classification gives a counterexample for $n \geq 3$.
\end{abstract}

\section{Introduction}

For $K / F$ a quadratic extension of $p$-adic fields, let $\sigma$ be the conjugation relative to this extension, and let $\eta_{K / F}$ be the character of $F^{*}$ with kernel being norms of $K^{*}$.

Let $\pi$ be a smooth irreducible representation of $\operatorname{GL}(n, K)$, let $\chi$ be a character of $F^{*}$, and let $m$ be dimension of the space of linear forms on $\pi$ 's space that transform by $\chi$ under $\operatorname{GL}(n, F)$ with respect to the action $(L, g) \mapsto L \circ \pi(g)$. By [Flicker 1991, Proposition 11], $m$ is known to be at most one. One says that $\pi$ is $\chi$-distinguished if $m=1$; one says $\pi$ is distinguished if it is 1-distinguished.

In this article, we give a description of distinguished principal series representations of $\mathrm{GL}(n, K)$.

The result, Theorem 3.4, is that the irreducible distinguished representations of the principal series of $\operatorname{GL}(n, K)$ are (up to isomorphism) those unitarily induced from a character $\chi=\left(\chi_{1}, \ldots, \chi_{n}\right)$ of the maximal torus of diagonal matrices such that there exists an $r \leq n / 2$ for which $\chi_{i+1}^{\sigma}=\chi_{i}^{-1}$ for $i=1,3, \ldots, 2 r-1$, and $\left.\chi_{i}\right|_{F^{*}}=1$ for $i>2 r$. For the quadratic extension $\mathbb{C} / \mathbb{R}$, it is known [Panichi 2001] that the analogous result is true for tempered representations.

For $n \geq 3$, this gives a counterexample (see Corollary 3.5) to a conjecture of Jacquet [Anandavardhanan 2005, Conjecture 1], which states that an irreducible

MSC2000: primary 22E50; secondary 22E35.

Keywords: distinguished representations, Jacquet's conjecture. 
representation $\pi$ of $\mathrm{GL}(n, K)$ with central character trivial on $F^{*}$ is isomorphic to $\check{\pi}^{\sigma}$ if and only if it is distinguished or $\eta_{K / F}$-distinguished (where $\eta_{K / F}$ is the character of order 2 of $F^{*}$, attached by local class field theory to the extension $K / F$ ). For discrete series representations, the conjecture is true, as proved in [Kable 2004].

Unitary irreducible distinguished principal series representations of $\operatorname{GL}(2, K)$ were described in [Hakim 1991]. The general case of distinguished irreducible principal series representations of $\operatorname{GL}(2, K)$ was treated in [Flicker and Hakim 1994]; we use this occasion to give a different proof. To do this, in Theorems 4.1 and 4.3, we extend a criterion of Hakim [1991, Theorem 4.1], which characterizes smooth unitary irreducible distinguished representations of $\operatorname{GL}(2, K)$ in terms of $\gamma$ factors at $1 / 2$, to all smooth irreducible distinguished representations of $\mathrm{GL}(2, K)$.

\section{Preliminaries}

Let $\phi$ be a group automorphism and $x$ an element of the group. We sometimes write $x^{\phi}$ instead of $\phi(x)$ and write $x^{-\phi}$ for the inverse of $x^{\phi}$. If $\phi=x \mapsto h^{-1} x h$ for $h$ in the group, then we may write $x^{\phi}$ as $x^{h}$.

Let $G$ be a locally compact totally disconnected group, and let $H$ be a closed subgroup of $G$.

We denote by $\Delta_{G}$ the module of $G$ given by the relation $d_{G}(g x)=\Delta_{G}(g) d_{G}(x)$, where $d_{G}$ is the right Haar measure on $G$.

Let $X$ be a locally closed subspace of $G$ with $H . X \subset X$. If $V$ is a complex vector space, we denote by $D(X, V)$ the space of smooth $V$-valued functions on $X$ with compact support (if $V=\mathbb{C}$, we simply write $D(X)$ ).

Let $\rho$ be a smooth representation of $H$ in a complex vector space $V_{\rho}$. We denote by $D\left(H \backslash X, \rho, V_{\rho}\right)$ the space of smooth $V_{\rho}$-valued functions $f$ on $X$, with compact support modulo $H$, such that $f(h x)=\rho(h) f(x)$ for $h \in H$ and $x \in X$ (if $\rho$ is a character, we simply write $D(H \backslash X, \rho))$.

We denote by $\operatorname{ind}_{H}^{G}(\rho)$ the representation induced by right translations of $G$ in $D\left(H \backslash G,\left(\Delta_{G} / \Delta_{H}\right)^{1 / 2} \rho, V_{\rho}\right)$.

Let $F$ be a nonarchimedean local field of characteristic zero, and let $K$ be a quadratic extension of $F$. We have $K=F(\delta)$ with $\delta^{2}$ in $F^{*}$.

We denote by $|\cdot|_{K}$ the absolute value on $K$.

We denote by $\sigma$ the nontrivial element of the Galois group $G(K / F)$ of $K$ over $F$, and we use the same letter to designate its action on $M_{n}(K)$.

We denote by $N_{K / F}$ the norm of the extension $K / F$, and we write $\eta_{K / F}$ for the nontrivial character of $F^{*}$ that is trivial on $N_{K / F}\left(K^{*}\right)$.

Whenever $G$ is an algebraic group defined over $F$, we denote by $G(K)$ its $K$-points and by $G(F)$ its $F$-points. 
We denote by $G_{n}$ the group GL( $\left.n\right)$, by $B_{n}$ its standard Borel subgroup, by $U_{n}$ its unipotent radical, and by $T_{n}$ the standard maximal split torus of diagonal matrices.

We denote by $S$ the space of matrices $M$ in $G_{n}(K)$ satisfying $M M^{\sigma}=1$.

Everything in this section is more or less contained in [Flicker 1992], but we give detailed proofs here for convenience of the reader.

Proposition 2.1 [Serre 1968, Chapter 10, Proposition 3]. The map $S_{n}: g \mapsto g^{\sigma} g^{-1}$ is a homeomorphism between $G_{n}(K) / G_{n}(F)$ and $S$.

Proposition 2.2. For its natural action on $S$, each orbit of $B_{n}(K)$ contains one and only one element of $\mathfrak{S}_{n}$ of order 2 or 1.

Proof. We begin with the following:

Lemma 2.3. Let $w$ be an element of $\mathfrak{S}_{n} \subset G_{n}(K)$ of order at most 2.

Let $\theta^{\prime}$ be the involution of $T_{n}(K)$ given by $t \mapsto w^{-1} t^{\sigma} w$. Then any $t \in T_{n}(K)$ with $t \theta^{\prime}(t)=1$ is of the form $a / \theta^{\prime}(a)$ for some $a \in T_{n}(K)$.

Proof of Lemma 2.3. There exists a $r \leq n / 2$ such that, up to conjugacy,

$$
w=(1,2)(3,4) \cdots(2 r-1,2 r) .
$$

We write $t=\operatorname{diag}\left(z_{1}, z_{1}^{\prime}, \ldots, z_{r}, z_{r}^{\prime}, z_{2 r+1}, \ldots, z_{n}\right)$. Hence for $i \leq r$, we have $z_{i} \sigma\left(z_{i}^{\prime}\right)=1$, and $z_{j} \sigma\left(z_{j}\right)=1$ for $j \geq 2 r+1$.

Hilbert's Theorem 90 says each $z_{j}$ for $j \geq 2 r+1$ is of the form $u_{j-2 r} / \sigma\left(u_{j-2 r}\right)$ for some $u_{j-2 r} \in K^{*}$.

We then take $a=\operatorname{diag}\left(z_{1}, 1, \ldots, z_{r}, 1, u_{1}, \ldots, u_{n-2 r}\right)$.

Lemma 2.4. Let $N$ be an algebraic connected unipotent group over $K$. Let $\theta$ be an involutive automorphism of $N(K)$. If $x \in N(K)$ satisfies $x \theta(x)=1_{N}$, then there is an $a \in N$ such that $x=\theta\left(a^{-1}\right) a$.

Proof of Lemma 2.4. The group $N(K)$ has a composition series $1_{N}=N_{0} \subset N_{1} \subset$ $\cdots \subset N_{n-1} \subset N_{n}=N(K)$ such that each quotient $N_{i+1} / N_{i}$ is isomorphic to $(K,+)$ and such that each commutator subgroup [N, $\left.N_{i+1}\right]$ is a subgroup of $N_{i}$.

Now we prove the lemma by induction on $n$. If $n=1$, then $N(K)$ is isomorphic to $(K,+)$, one concludes taking $a=x / 2$. For the induction step, suppose the lemma is true for every $N(K)$ of length $n$. Let $N(K)$ be of length $n+1$.

By the induction hypothesis, one gets that there exists an element in $h \in N_{1}$ and an element $u$ in $N(K)$ such that $x=\theta\left(u^{-1}\right) u h$. Here $h$ lies in the center of $N(K)$, because $\left[N(K), N_{1}\right]=1_{N}$.

Because $x \theta(x)=1$, we get $h \theta(h)=1$. By the induction hypothesis again, we get $h=\theta\left(b^{-1}\right) b$ for $b \in N_{1}$. We then take $a=u b$.

We return to the proof of Proposition 2.2.

For $w$ in $\mathfrak{S}_{n}$, denote by $U_{w}$ the subgroup of $U_{n}$ generated by the elementary subgroups $U_{\alpha}$, with $\alpha$ positive and $w \alpha$ negative; denote by $U_{w}^{\prime}$ the subgroup of $U_{n}$ 
generated by the elementary subgroups $U_{\alpha}$, with $\alpha$ positive and $w \alpha$ positive. Then $U_{n}=U_{w}^{\prime} U_{w}$.

Let $s$ be in $S$. According to Bruhat's decomposition, there is a $w$ in $\mathfrak{S}_{n}$, an $a$ in $T_{n}(K)$, an $n_{1}$ in $U_{n}(K)$, and an $n_{2}^{+}$in $U_{w}$ such that $s=n_{1} a w n_{2}^{+}$; this decomposition is unique.

Then $s=s^{-\sigma}=\left(n_{2}^{+}\right)^{-\sigma} w^{-1} a^{-\sigma} n_{1}^{-\sigma}$. Thus we have $a w=(a w)^{-\sigma}$, that is, $w^{2}=1$ and $a^{w}=a^{-\sigma}$.

Now we write $n_{1}^{-\sigma}=u^{-} u^{+}$with $u^{-} \in U_{w}^{\prime}$ and $u^{+} \in U_{w}$. Then, comparing $s$ and $s^{-\sigma}$, we see $u^{+}$must be equal to $n_{2}{ }^{+}$. Hence $s=n_{1} a w\left(u^{-}\right)^{-1} n_{1}{ }^{-\sigma}$; thus we suppose $s=a w n$, with $n$ in $U_{w}^{\prime}$.

From $s=s^{-\sigma}$, we have the relation $\operatorname{awn}(a w)^{-1}=n^{-\sigma}$. Applying $\sigma$ on each side, this becomes $(a w)^{-1} n^{\sigma} a w=n^{-1}$.

But $\theta: u \mapsto(a w)^{-1} u^{\sigma} a w$ is an involutive automorphism of $U_{w}^{\prime}$; hence from Lemma 2.4, there is a $u^{\prime}$ in $U_{w}^{\prime}$ such that $n=\theta\left(u^{-1}\right) u$. This gives $s=u^{-\sigma} a w u$, so that we suppose $s=a w$. Again $w a^{\sigma} w=a^{-1}$, and applying Lemma 2.3 to $\theta^{\prime}: x \mapsto w x^{\sigma} w$, we deduce that $a$ is of the form $y \theta^{\prime}\left(y^{-1}\right)$, and $s=y w y^{-\sigma}$.

Let $u \in M_{2}(K)$ equal $\left(\begin{array}{cc}1 & -\delta \\ 1 & \delta\end{array}\right)$. We have $S_{2}(u)=\left(\begin{array}{ll}0 & 1 \\ 1 & 0\end{array}\right)$; see Proposition 2.1.

We note for further use (in the proof of Proposition 3.3) that if we define the subgroup $\widetilde{T}:=\left\{\left(\operatorname{diag}\left(z, z^{\sigma}\right) \in G_{2}(K) \mid z \in K^{*}\right\}\right.$, then

$$
u^{-1} \tilde{T} u=T=\left\{\left(\begin{array}{cc}
x & \delta^{2} y \\
y & x
\end{array}\right) \in G_{2}(F) \mid x, y \in F\right\} .
$$

For $r \leq n / 2$, we denote by $U_{r}$ the $n \times n$ matrix given by the block decomposition $\operatorname{diag}\left(u, \ldots, u, I_{n-2 r}\right)$.

For $w$ an element of $\mathfrak{S}_{n}$ naturally injected in $G_{n}(K)$, we write $U_{r}^{w}=w^{-1} U_{r} w$.

Corollary 2.5. The elements $U_{r}^{w}$ for $0 \leq r \leq n / 2$, and $w \in \mathfrak{S}_{n}$ give a complete set of representatives of classes of $B_{n}(K) \backslash G_{n}(K) / G_{n}(F)$.

Let $G_{n}=\bigsqcup_{w \in \mathfrak{S}_{n}} B_{n} w B_{n}$ be the Bruhat decomposition of $G_{n}$. We call a doubleclass $B w B$ a Bruhat cell.

Lemma 2.6. One can order the Bruhat cells $C_{1}, C_{2}, \ldots, C_{n}$ ! so that for every $1 \leq i \leq n$ !, the cell $C_{i}$ is closed in $G_{n}-\bigsqcup_{k=1}^{i-1} C_{i}$.

Proof. Choose $C_{1}=B_{n}$. It is closed in $G_{n}$. Now let $w_{2}$ be an element of $\mathfrak{S}_{n}-\mathrm{Id}$ with minimal length. Then from [Springer 1998, 8.5.5], the Bruhat cell $B w_{2} B$ is closed in $G_{n}-B_{n}$ in the Zariski topology; hence in the $p$-adic topology, we may take this cell to be $C_{2}$. We conclude by repeating this process.

Corollary 2.7. One can order the classes $A_{1}, \ldots, A_{t}$ of $B_{n}(K) \backslash G_{n}(K) / G_{n}(F)$ so that $A_{i}$ is closed in $G_{n}(K)-\bigsqcup_{k=1}^{i-1} A_{i}$. 
Proof. From the proof of Proposition 2.2, we know that if $C$ is a Bruhat cell of $G_{n}$, then $S_{n} \cap C$ is either empty or it corresponds through the homeomorphism $S_{n}$ to a class $A$ of $B_{n}(K) \backslash G_{n}(K) / G_{n}(F)$. The conclusion then follows from the previous lemma.

Corollary 2.8. Each $A_{i}$ is locally closed in $G_{n}(K)$ in the Zariski topology.

Lemma 2.9. Let $G, H, X$, and $\left(\rho, V_{\rho}\right)$ be as in the beginning of the section. The map

$$
\Phi: D(X) \otimes V_{\rho} \rightarrow D\left(H \backslash X, \rho, V_{\rho}\right), \quad f \otimes v \mapsto\left(x \mapsto \int_{H} f(h x) \rho\left(h^{-1}\right) v d h\right)
$$

is surjective.

Proof. Let $v \in V_{\rho}$. Let $U$ be an open subset of $G$ intersecting $X$ and small enough for $h \mapsto \rho(h) v$ to be trivial on $H \cap U U^{-1}$. Let $f^{\prime}$ be the function with support in $H(X \cap U)$ defined by $h x \mapsto \rho(h) v$. Such functions generate $D\left(H \backslash X, \rho, V_{\rho}\right)$ as a vector space.

Now let $f$ be the function of $D\left(X, V_{\rho}\right)$ defined by $x \mapsto 1_{U \cap X}(x) v$. Then $\Phi(f)$ is a multiple of $f^{\prime}$.

But for $x$ in $U \cap X, \Phi(f)(x)=\int_{H} \rho\left(h^{-1}\right) f(h x) d h$ because $h \mapsto \rho(h) v$ is trivial on $H \cap U U^{-1}$. Also $h \mapsto f(h x)$ is a positive function that multiplies $v$, and $f(x)=V$. Thus $F(f)(x)$ is $v$ multiplied by a strictly positive scalar.

Corollary 2.10. Let $Y$ be an $H$-stable closed subset of $X$. Then the restriction map from $D\left(H \backslash X, \rho, V_{\rho}\right)$ to $D\left(H \backslash Y, \rho, V_{\rho}\right)$ is surjective.

Proof. This is a consequence of the known surjectivity of the restriction map from $D(X)$ to $D(Y)$, which implies the surjectivity of the restriction from $D\left(X, V_{\rho}\right)$ to $D\left(Y, V_{\rho}\right)$, and of the commutativity of the diagram

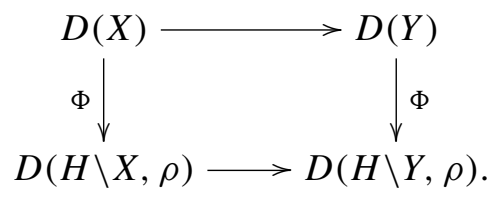

\section{Distinguished principal series}

If $\pi$ is a smooth representation of $G_{n}(K)$ on the space $V_{\pi}$ and $\chi$ is a character of $F^{*}$, we say that $\pi$ is $\chi$-distinguished if there exists on $V_{\pi}$ a nonzero linear form $L$ such that $L(\pi(g) v)=\chi(\operatorname{det}(g)) L(v)$ whenever $g$ is in $G_{n}(F)$ and $v$ belongs to $V_{\pi}$. If $\chi$ is trivial, we simply say that $\pi$ is distinguished.

We first recall the following:

Theorem 3.1 [Flicker 1991, Proposition 12]. Let $\pi$ be a smooth irreducible distinguished representation of $G_{n}(K)$. Then $\pi^{\sigma} \simeq \check{\pi}$. 
Let $\chi_{1}, \ldots, \chi_{n}$ be $n$ characters of $K^{*}$, with none of their quotients equal to $|\cdot|_{K}$. We denote by $\chi$ the character of $b \in B_{n}(K)$ defined by $\chi(b)=\chi_{1}\left(b_{1}\right) \cdots \chi_{n}\left(b_{n}\right)$, where the $b_{i}$ are the diagonal entries of $b$.

We denote by $\pi(\chi)$ the representation of $G_{n}(K)$ by right translation on the space of functions $D\left(B_{n}(K) \backslash G_{n}(K), \Delta_{B_{n}}^{-1 / 2} \chi\right)$. This representation is smooth and irreducible; we call it the principal series attached to $\chi$. For a smooth representation $\pi$ of $G_{n}(K)$, we denote by $\check{\pi}$ its smooth contragredient.

Lemma 3.2 [Flicker 1992, Proposition 26]. Let $\bar{m}=\left(m_{1}, \ldots, m_{l}\right)$ be a partition of a positive integer $m$, let $P_{\bar{m}}$ be the corresponding standard parabolic subgroup, and for each $1 \leq i \leq l$, let $\pi_{i}$ be a smooth distinguished representation of $G_{m_{i}}(K)$. Then

$$
\pi_{1} \times \cdots \times \pi_{l}=\operatorname{ind}_{P_{\bar{m}}(K)}^{G_{m}(K)}\left(\Delta_{P_{\bar{m}}(K)}^{-1 / 2}\left(\pi_{1} \otimes \cdots \otimes \pi_{l}\right)\right)
$$

is distinguished.

We now come to the principal result:

Proposition 3.3. Let $\chi=\left(\chi_{1}, \ldots, \chi_{n}\right)$ be a character of $B_{n}(K)$ with none of the characters $\chi_{i} / \chi_{j}$ equal to $|\cdot|_{K}$. Suppose that the principal series representation $\pi(\chi)$ is distinguished. Then there exists a reordering of the $\chi_{i}$ and an $r \leq n / 2$ satisfying $\chi_{i+1}^{\sigma}=\chi_{i}^{-1}$ for $i=1,3, \ldots, 2 r-1$ and $\left.\chi_{i}\right|_{F^{*}}=1$ for $i>2 r$.

Proof. We write $B=B_{n}(K)$ and $G=G_{n}(K)$. From Corollaries 2.7 and 2.10, we have the following exact sequence of smooth $G_{n}(F)$-modules:

$$
D\left(B \backslash G-A_{1}, \Delta_{B}^{-1 / 2} \chi\right) \hookrightarrow D\left(B \backslash G, \Delta_{B}^{-1 / 2} \chi\right) \rightarrow D\left(B \backslash A_{1}, \Delta_{B}^{-1 / 2} \chi\right) .
$$

Hence there is a nonzero distinguished linear form either on $D\left(B \backslash A_{1}, \Delta_{B}^{-1 / 2} \chi\right)$, or on $D\left(B \backslash G-A_{1}, \Delta_{B}^{-1 / 2} \chi\right)$.

In the second case, we have the exact sequence

$$
D\left(B \backslash G-A_{1} \sqcup A_{2}, \Delta_{B}^{-1 / 2} \chi\right) \hookrightarrow D\left(B \backslash G-A_{1}, \Delta_{B}^{-1 / 2} \chi\right) \rightarrow D\left(B \backslash A_{2}, \Delta_{B}^{-1 / 2} \chi\right) .
$$

Repeating the process, we deduce the existence of a nonzero distinguished linear form on one of the spaces $D\left(B \backslash A_{i}, \Delta_{B}^{-1 / 2} \chi\right)$.

By Corollary 2.5, we may choose $w$ in $S_{n}$ and $r \leq n / 2$ with $A_{i}=B U_{r}^{w} G_{n}(F)$. The application $f \mapsto\left(x \mapsto f\left(U_{r}^{w} x\right)\right)$ gives an isomorphism of $G_{n}(F)$-modules between

$$
D\left(B \backslash A_{i}, \Delta_{B}^{-1 / 2} \chi\right) \quad \text { and } \quad D\left(U_{r}^{-w} B U_{r}^{w} \cap G_{n}(F) \backslash G_{n}(F), \Delta^{\prime} \chi^{\prime}\right),
$$

where $\Delta^{\prime}(x)=\Delta_{B}^{-1 / 2}\left(U_{r}^{w} x U_{r}^{-w}\right)$ and $\chi^{\prime}(x)=\chi\left(U_{r}^{w} x U_{r}^{-w}\right)$.

Now there exists a nonzero $G_{n}(F)$-invariant linear form on

$$
D\left(U_{r}^{-w} B U_{r}^{w} \cap G_{n}(F) \backslash G_{n}(F), \Delta^{\prime} \chi^{\prime}\right)
$$


if and only if $\Delta^{\prime} \chi^{\prime}$ is equal to the inverse of the module of $U_{r}^{-w} B U_{r}^{w} \cap G_{n}(F)$; see [Bushnell and Henniart 2006, Chapter 1, Proposition 3.4]. From this we deduce that $\chi^{\prime}$ is positive on $U_{r}^{-w} B U_{r}^{w} \cap G_{n}(F)$ or equivalently $\chi$ is positive on $B \cap U_{r}^{w} G_{n}(F) U_{r}^{-w}$.

Let $\bar{T}_{r}$ be the $F$-torus of matrices of the form

$$
\operatorname{diag}\left(z_{1}, z_{1}^{\sigma}, \ldots, z_{r}, z_{r}^{\sigma}, x_{1}, \ldots, x_{t}\right)
$$

where $2 r+t=n, z_{i} \in K^{*}$, and $x_{i} \in F^{*}$. Then $\bar{T}_{r}^{w} \subset B \cap U_{r}^{w} G_{n}(F) U_{r}^{-w}$, so that $\chi$ must be positive on $\bar{T}_{r}^{w}$.

If $\chi$ is unitary, then $\chi$ is trivial on $\bar{T}_{r}^{w}$, and $\pi(\chi)$ is of the desired form.

For the general case, we deduce from Theorem 3.1, that there exist three integers $p \geq 0, q \geq 0$, and $s \geq 0$ such that up to reordering the $\chi_{i}$ are as follows: For $1 \leq i \leq p$, we have $\chi_{2 i}=\chi_{2 i-1}^{-\sigma}$. For $1 \leq k \leq q$, we have $\left.\chi_{2 p+k}\right|_{F^{*}}=1$, and these $\chi_{2 p+k}$ are distinct (meaning $\chi_{2 p+k} \neq \chi_{2 p+k^{\prime}}^{-\sigma}$ for $k \neq k^{\prime}$ ). For $1 \leq j \leq s$, we have $\left.\chi_{2 p+q+j}\right|_{F^{*}}=\eta_{K / F}$, and these $\chi_{2 p+q+j}$ are distinct.

We write $\mu_{k}=\chi_{2 p+k}$ for $q \geq k \geq 1$ and $v_{k}^{\prime}=\chi_{2 p+q+k^{\prime}}$ for $s \geq k^{\prime} \geq 1$.

We will show that if such a character $\chi$ is positive on a conjugate of $\bar{T}_{r}$ by an element of $S_{n}$, then $s=0$.

Suppose $v_{1}$ appears. Then either $v_{1}$ is positive on $F^{*}$, which is not possible, or it is paired with another $\chi_{i}$, and $\left(v_{1}, \chi_{i}\right)$ is positive on elements $\left(z, z^{\sigma}\right)$ for $z$ in $K^{*}$.

Suppose $\chi_{i}=v_{j}$ for some $j \neq 1$. Then $\left(v_{1}, \chi_{i}\right)$ is unitary, so it must be trivial on pairs $\left(z, z^{\sigma}\right)$, which implies $v_{1}=v_{j}{ }^{-\sigma}=v_{j}$, which is absurd.

The character $\chi_{i}$ cannot be of the form $\mu_{j}$, because this would imply $\left.v_{1}\right|_{F^{*}}=1$.

Suppose finally that $i \leq 2 p$. In this case $v_{1}{ }^{-\sigma}=v_{1}$ must be the unitary part of $\chi_{i}$ because of the positivity of $\left(v_{1}, \chi_{i}\right)$ on the pairs $\left(z, z^{\sigma}\right)$.

But $\chi_{i}^{-\sigma}$ also appears and is not trivial on $F^{*}$. Hence it must be paired with another character $\chi_{j}$ with $j \leq 2 p$ and $j \neq i$ such that $\left(\chi_{i}^{-\sigma}, \chi_{j}\right)$ is positive on the elements $\left(z, z^{\sigma}\right)$ for $z$ in $K^{*}$. This implies that $\chi_{j}$ has unitary part $v_{1}^{-\sigma}=v_{1}$. The character $\chi_{j}$ cannot be a $\mu_{k}$ because of its unitary part.

If it is a $\chi_{k}$ with $k \leq 2 p$, we consider $\chi_{k}^{-\sigma}$ again.

By repeating the process long enough, we can suppose that $\chi_{j}$ is of the form $v_{k}$ with $k \neq 1$. Taking unitary parts, we see that $v_{k}=v_{1}^{-\sigma}=v_{1}$, which is in contradiction with the fact that the $v_{i}$ are all different. We conclude that $s=0$.

Theorem 3.4. Let $\chi=\left(\chi_{1}, \ldots, \chi_{n}\right)$ be a character of $T_{n}(K)$. Then the principal series representation $\pi(\chi)$ is distinguished if and only if there exists an $r \leq n / 2$ such that $\chi_{i+1}^{\sigma}=\chi_{i}^{-1}$ for $i=1,3, \ldots, 2 r-1$ and $\left.\chi_{i}\right|_{F^{*}}=1$ for $i>2 r$.

Proof. There is one implication left. 
Suppose $\chi$ is of the desired form. Then $\pi(\chi)$ is parabolically (unitarily) induced from representations of the type $\pi\left(\chi_{i}, \chi_{i}^{-\sigma}\right)$ of $G_{2}(K)$ and from distinguished characters of $K^{*}$.

Hence by Lemma 3.2 we need only show that the representations $\pi\left(\chi_{i}, \chi_{i}^{-\sigma}\right)$ are distinguished. But this is just Corollary 4.2 of the next section.

This gives a counterexample to a conjecture of Jacquet [Anandavardhanan 2005, Conjecture 1], which asserts that if an irreducible admissible representation $\pi$ of $G_{n}(K)$ is such that $\check{\pi}$ is isomorphic to $\pi^{\sigma}$, then it is distinguished if $n$ is odd, and is distinguished or $\eta_{K / F}$-distinguished if $n$ is even.

Corollary 3.5. For $n \geq 3$, there exists a smooth irreducible representation $\pi$ of $G_{n}(K)$, with central character trivial on $F^{*}$, that is neither distinguished nor $\eta_{K / F}$-distinguished but whose smooth contragredient $\check{\pi}$ is isomorphic to $\pi^{\sigma}$.

Proof. Take $\chi_{1}, \ldots, \chi_{n}$, all different, such that $\left.\chi_{1}\right|_{F^{*}}=\left.\chi_{2}\right|_{F^{*}}=\eta_{K / F}$, and $\left.\chi_{j}\right|_{F^{*}}=1$ for $3 \leq j \leq n$. Because each $\chi_{i}$ has trivial restriction to $N_{K / F}\left(K^{*}\right)$, it is equal to $\chi_{i}^{-\sigma}$; hence $\check{\pi}$ is isomorphic to $\pi^{\sigma}$. Another consequence is that if $k$ and $l$ are two distinct integers between 1 and $n$, then $\chi_{k} \neq \chi_{l}^{-\sigma}$, because we assumed the $\chi_{i}$ are all different.

Then it follows from Theorem 3.4 that $\pi=\pi\left(\chi_{1}, \ldots, \chi_{n}\right)$ is neither distinguished nor $\eta_{K / F}$-distinguished, but clearly the central character of $\pi$ is trivial on $F^{*}$, and $\check{\pi}$ is isomorphic to $\pi^{\sigma}$.

\section{Distinguishability and gamma factors for GL(2)}

In this section we generalize to smooth infinite dimensional irreducible representations of $G_{2}(K)$ a criterion of Hakim [1991, Theorem 4.1], which characterizes smooth unitary irreducible distinguished representations of $G_{2}(K)$. In the proof of that theorem, Hakim deals with unitary representations so that the integrals of Kirillov functions on $F^{*}$ with respect to a Haar measure of $F^{*}$ converge. We bypass the convergence problems using [Jacquet and Langlands 1970, Proposition 2.9 of Chapter 1].

We denote $M(K)$ by the mirabolic subgroup of $G_{2}(K)$ of matrices of the form $\left(\begin{array}{ll}a & x \\ 0 & 1\end{array}\right)$ with $a$ in $K^{*}$ and $x$ in $K$, and by $M(F)$ its intersection with $G_{2}(F)$. We let $w=\left(\begin{array}{rr}0 & -1 \\ 1 & 0\end{array}\right)$.

Let $\pi$ be a smooth infinite dimensional irreducible representation of $G_{2}(K)$. It is known that $\pi$ is generic (see for example [Zelevinsky 1980]). Let $K(\pi, \psi)$ be its Kirillov model corresponding to $\psi$ [Jacquet and Langlands 1970, Theorem 2.13]. This model contains the subspace $D\left(K^{*}\right)$ of functions with compact support on the group $K^{*}$. If $\phi$ belongs to $K(\pi, \psi)$ and $x$ belongs to $K$, then $\phi-\pi\left(\begin{array}{ll}1 & x \\ 0 & 1\end{array}\right) \phi$ belongs to $D\left(K^{*}\right)$ [Jacquet and Langlands 1970, Chapter 1, Proposition 2.9]. From this follows that $K(\pi, \psi)=D\left(K^{*}\right)+\pi(w) D\left(K^{*}\right)$. 
We now recall a consequence of the functional equation at $1 / 2$ for Kirillov representations [Bump 1997, Section 4.7].

For all $\phi$ in $K(\pi, \psi)$ and $\chi$ a character of $K^{*}$, we have

$$
\int_{K^{*}} \pi(w) \phi(x)\left(c_{\pi} \chi\right)^{-1}(x) d^{*} x=\gamma(\pi \otimes \chi, \psi) \int_{K^{*}} \phi(x) \chi(x) d^{*} x
$$

whenever both sides converge absolutely, where $d^{*} x$ is a Haar measure on $K^{*}$ and $c_{\pi}$ is the central character of $\pi$.

Theorem 4.1. Let $\pi$ be a smooth irreducible representation of $G_{2}(K)$ of infinite dimension with central character trivial on $F^{*}$, and let $\psi$ be a nontrivial character of $K$ trivial on $F$. If $\gamma(\pi \otimes \chi, \psi)=1$ for every character $\chi$ of $K^{*}$ trivial on $F^{*}$, then $\pi$ is distinguished.

Proof. In fact, using a Fourier inversion in the functional equation (1) and the change of variable $x \mapsto x^{-1}$, we deduce that

$$
c_{\pi}(x) \int_{F^{*}} \pi(w) \phi\left(t x^{-1}\right) d^{*} t=\int_{F^{*}} \phi(t x) d^{*} t \quad \text { for all } \phi \in D\left(K^{*}\right) \cap \pi(w) D\left(K^{*}\right),
$$

where $d^{*} t$ is a Haar measure on $F^{*}$. For $x=1$, this gives

$$
\int_{F^{*}} \pi(w) \phi(t) d^{*} t=\int_{F^{*}} \phi(t) d^{*} t .
$$

Now we define on $K(\pi, \psi)$ a linear form $\lambda$ by

$$
\lambda\left(\phi_{1}+\pi(w) \phi_{2}\right)=\int_{F^{*}} \phi_{1}(t) d^{*} t+\int_{F^{*}} \phi_{2}(t) d^{*} t \quad \text { for } \phi_{1}, \phi_{2} \in D\left(K^{*}\right) .
$$

This is well defined by because of the previous equality and the fact that $K(\pi, \psi)=$ $D\left(K^{*}\right)+\pi(w) D\left(K^{*}\right)$.

It is clear that $\lambda$ is $w$-invariant. Since the central character of $\pi$ is trivial on $F^{*}$, $\lambda$ is also $F^{*}$-invariant. Because $\mathrm{GL}_{2}(F)$ is generated by $M(F)$, its center, and $w$, it remains to show that $\lambda$ is $M(F)$-invariant.

Since $\psi$ is trivial on $F$, we have $\lambda(\pi(m) \phi)=\lambda(\phi)$ if $\phi \in D\left(K^{*}\right)$ and $m \in M(F)$.

Now if $\phi=\pi(w) \phi_{2} \in \pi(w) D\left(K^{*}\right)$ and if $a$ belongs to $F^{*}$, then

$$
\pi\left(\begin{array}{ll}
a & 0 \\
0 & 1
\end{array}\right) \pi(w) \phi_{2}=\pi(w) \pi\left(\begin{array}{ll}
1 & 0 \\
0 & a
\end{array}\right) \phi_{2}=\pi(w) \pi\left(\begin{array}{cc}
a^{-1} & 0 \\
0 & 1
\end{array}\right) \phi_{2}
$$

because the central character of $\pi$ is trivial on $F^{*}$, and $\lambda\left(\pi\left(\begin{array}{ll}a & 0 \\ 0 & 1\end{array}\right) \phi\right)=\lambda(\phi)$.

If $x \in F$, then $\pi\left(\begin{array}{ll}1 & x \\ 0 & 1\end{array}\right) \phi-\phi$ is a function in $D\left(K^{*}\right)$, which vanishes on $F^{*}$. Hence $\lambda \pi\left(\left(\begin{array}{ll}1 & x \\ 0 & 1\end{array}\right) \phi-\phi\right)=0$.

Eventually $\lambda$ is $M(F)$-invariant and hence $G_{2}(F)$-invariant; it is clear that its restriction to $D\left(K^{*}\right)$ is nonzero.

Corollary 4.2. Let $\mu$ be a character of $K^{*}$. Then $\pi\left(\mu, \mu^{-\sigma}\right)$ is distinguished. 
Proof. First note that the central character $\mu \mu^{-\sigma}$ of $\pi\left(\mu, \mu^{-\sigma}\right)$ is trivial on $F^{*}$.

Now let $\chi$ be a character of $K^{*} / F^{*}$. Then

$$
\gamma\left(\pi\left(\mu, \mu^{-\sigma}\right) \otimes \chi, \psi\right)=\gamma(\mu \chi, \psi) \gamma\left(\mu^{-\sigma} \chi, \psi\right)=\gamma(\mu \chi, \psi) \gamma\left(\mu^{-1} \chi^{\sigma}, \psi^{\sigma}\right),
$$

and since $\left.\psi\right|_{F}=1$ and $\left.\chi\right|_{F^{*}}=1$, we have $\psi^{\sigma}=\psi^{-1}$ and $\chi^{\sigma}=\chi^{-1}$. Thus $\gamma\left(\pi\left(\chi, \chi^{-\sigma}\right), \psi\right)=\gamma(\mu \chi, \psi) \gamma\left(\mu^{-1} \chi^{-1}, \psi^{-1}\right)=1$. The conclusion then follows from Theorem 4.1.

Using [Aizenbud and Gourevitch 2007, Theorem 1.2], Theorem 4.1's converse is also true:

Theorem 4.3. Let $\pi$ be a smooth irreducible representation of infinite dimension of $G_{2}(K)$ with central character trivial on $F^{*}$, and let $\psi$ be a nontrivial character of $K / F$. Then $\pi$ is distinguished if and only if $\gamma(\pi \otimes \chi, \psi)=1$ for every character $\chi$ of $K^{*}$ that is trivial on $F^{*}$.

Proof. It suffices to show that if $\pi$ is a smooth irreducible distinguished representation of infinite dimension of $G_{2}(K)$ and $\psi$ is a nontrivial character of $K / F$, then $\gamma(\pi, \psi)=1$. Suppose $\lambda$ is a nonzero $G_{2}(F)$-invariant linear form on $K(\pi, \psi)$. The proof of the corollary to [Hakim 1991, Proposition 3.3] shows that $\lambda(\phi)$ is equal to $\int_{F^{*}} \phi(t) d^{*} t$ for $\phi$ in $D\left(K^{*}\right)$. Hence for any function $\phi$ in $D\left(K^{*}\right) \cap \pi(w) D\left(K^{*}\right)$, we must have $\int_{F^{*}} \phi(t) d^{*} t=\int_{F^{*}} \pi(w) \phi(t) d^{*} t$.

From this we deduce that for any function $\phi$ in $D\left(K^{*}\right) \cap \pi(w) D\left(K^{*}\right)$, we have

$$
\begin{aligned}
\int_{K^{*}} \pi(w) \phi(x) c_{\pi}^{-1}(x) d^{*} x & =\int_{K^{*} / F^{*}} c_{\pi}^{-1}(a) \int_{F^{*}} \pi(w) \phi(t a) d^{*} t d a \\
& =\int_{K^{*} / F^{*}} c_{\pi}^{-1}(a) \int_{F^{*}} \pi\left(\begin{array}{cc}
a & 0 \\
0 & 1
\end{array}\right) \pi(w) \phi(t) d^{*} t d a \\
& =\int_{K^{*} / F^{*}} c_{\pi}^{-1}(a) \int_{F^{*}} \pi(w) c_{\pi}(a) \pi\left(\begin{array}{cc}
a^{-1} & 0 \\
0 & 1
\end{array}\right) \phi(t) d^{*} t d a \\
& =\int_{K^{*} / F^{*}} \int_{F^{*}} \pi\left(\begin{array}{cc}
a^{-1} & 0 \\
0 & 1
\end{array}\right) \phi(t) d^{*} t d a \\
& =\int_{K^{*} / F^{*}} \int_{F^{*}} \phi\left(t a^{-1}\right) d^{*} t d a \\
& =\int_{K^{*} / F^{*}} \int_{F^{*}} \phi(t a) d^{*} t d a=\int_{K^{*}} \phi(x) d^{*} x .
\end{aligned}
$$

This implies that either $\gamma(\pi, \psi)$ is equal to one or $\int_{K^{*}} \phi(x) d^{*} x$ is equal to zero on $D\left(K^{*}\right) \cap \pi(w) D\left(K^{*}\right)$. The latter cannot be the case, because we could then define two independent $K^{*}$-invariant linear forms on $K(\pi, \psi)=D\left(K^{*}\right)+\pi(w) D\left(K^{*}\right)$ given by $\phi_{1}+\pi(w) \phi_{2} \mapsto \int_{K^{*}} \phi_{1}(x) d^{*} x$ and $\phi_{1}+\pi(w) \phi_{2} \mapsto \int_{K^{*}} \phi_{2}(x) d^{*} x$, which contradicts [Aizenbud and Gourevitch 2007, Theorem 1.2]. 


\section{Acknowledgments}

I would like to thank Corinne Blondel and Paul Gérardin for many helpful comments and suggestions.

\section{References}

[Aizenbud and Gourevitch 2007] A. Aizenbud and D. Gourevitch, "A proof of the multiplicity one conjecture for GL $(n)$ in GL $(n+1)$ ", preprint, 2007. arXiv 0707.2363v2

[Anandavardhanan 2005] U. K. Anandavardhanan, "Distinguished non-Archimedean representations", pp. 183-192 in Algebra and number theory (Hyderabad, 2003), edited by R. Tandon, Hindustan Book Agency, Delhi, 2005. MR 2006k:22013 Zbl 1079.22013

[Bump 1997] D. Bump, Automorphic forms and representations, Cambridge Studies in Advanced Mathematics 55, Cambridge University Press, 1997. MR 97k:11080 Zbl 0868.11022

[Bushnell and Henniart 2006] C. J. Bushnell and G. Henniart, The local Langlands conjecture for GL(2), Grundlehren series 335, Springer, Berlin, 2006. MR 2007m:22013 Zbl 1100.11041

[Flicker 1991] Y. Z. Flicker, "On distinguished representations", J. Reine Angew. Math. 418 (1991), 139-172. MR 92i:22019 Zbl 0725.11026

[Flicker 1992] Y. Z. Flicker, "Distinguished representations and a Fourier summation formula", Bull. Soc. Math. France 120:4 (1992), 413-465. MR 93j:22033 Zbl 0778.11030

[Flicker and Hakim 1994] Y. Z. Flicker and J. L. Hakim, "Quaternionic distinguished representations", Amer. J. Math. 116:3 (1994), 683-736. MR 95i:22028 Zbl 0837.11030

[Hakim 1991] J. Hakim, "Distinguished p-adic representations", Duke Math. J. 62:1 (1991), 1-22. MR 92c:22037 Zbl 0724.22016

[Jacquet and Langlands 1970] H. Jacquet and R. P. Langlands, Automorphic forms on GL(2), Lecture Notes in Mathematics 114, Springer, Berlin, 1970. MR 53 \#5481 Zbl 0236.12010

[Kable 2004] A. C. Kable, "Asai $L$-functions and Jacquet's conjecture", Amer. J. Math. 126:4 (2004), 789-820. MR 2005g:11083 Zbl 1061.11023

[Panichi 2001] M.-N. Panichi, Caractérisations du spectre tempéré de $\mathrm{GL}_{n}(\mathbb{C}) / \mathrm{GL}_{n}(\mathbb{R})$, thesis, Université de Paris 7, 2001.

[Serre 1968] J.-P. Serre, Corps locaux, 2nd ed., Publications de l'Université de Nancago 8, Hermann, Paris, 1968. MR 50 \#7096 Zbl 0137.02601

[Springer 1998] T. A. Springer, Linear algebraic groups, 2nd ed., Progress in Math. 9, Birkhäuser, Boston, 1998. MR 99h:20075 Zbl 0927.20024

[Zelevinsky 1980] A. V. Zelevinsky, "Induced representations of reductive p-adic groups, II: On irreducible representations of GL(n)", Ann. Scient. École Norm. Sup. (4) 13:2 (1980), 165-210. MR 83g:22012 Zbl 0441.22014

Received August 2, 2008.

\section{NADIR MATRINGE}

INSTITUT DE MATHÉMATIQUES DE JUSSIEU

175, RUE DU CHEVALERET

75013 PARIS

FRANCE

matringe@math.jussieu.fr 